\title{
Air Pollution Exposure Monitoring among Pregnant Women with and without Asthma
}

\author{
Sandie Ha ${ }^{1, *}$, Carrie Nobles ${ }^{2}$, Jenna Kanner ${ }^{2}{ }^{-}$, Seth Sherman ${ }^{3}{ }^{(D)}$, Seung-Hyun Cho ${ }^{4}$, \\ Neil Perkins ${ }^{2}$, Andrew Williams ${ }^{5}$, William Grobman ${ }^{6}$, Joseph Biggio ${ }^{7}$, Akila Subramaniam ${ }^{8}$ (D, \\ Marion Ouidir ${ }^{2} \mathbb{D}$, Zhen Chen ${ }^{2}$ and Pauline Mendola ${ }^{2} \mathbb{B}$ \\ 1 Department of Public Health, Health Sciences Research Institute, College of Social Sciences, Humanities, \\ and Arts, University of California, Merced, CA 95343, USA \\ 2 Division of Intramural Population Health Research, Eunice Kennedy Shriver National Institute of Child \\ Health and Human Development, Bethesda, MD 20892, USA; cnobles@umass.edu (C.N.); \\ jenna.kanner@gmail.com (J.K.); perkinsn@mail.nih.gov (N.P.); marion.ouidir@nih.gov (M.O.); \\ chenzhe@mail.nih.gov (Z.C.); pauline.mendola@nih.gov (P.M.) \\ 3 The Emmes Company, Rockville, MD 20850, USA; ssherman@emmes.com \\ 4 RTI International, Research Triangle Park, NC 27709, USA; scho@rti.org \\ 5 School of Medicine \& Health Sciences, University of North Dakota, Grand Forks, ND 58202, USA; \\ andrew.d.williams@und.edu \\ 6 Feinberg School of Medicine, Northwestern University, Chicago, IL 60611, USA; \\ w-grobman@northwestern.edu \\ 7 Ochsner Health System, New Orleans, LA 70115, USA; joseph.biggio@ochsner.org \\ 8 Department of Obstetrics \& Gynecology, University of Alabama at Birmingham, Birmingham, AL 35294, \\ USA; asubramaniam@uabmc.edu \\ * Correspondence: sha55@ucmerced.edu; Tel.: +1-209-228-3615
}

Received: 29 April 2020; Accepted: 30 June 2020; Published: 7 July 2020

Abstract: Background: We monitored exposure to fine particulates $\left(\mathrm{PM}_{2.5}\right)$, ozone, nitrogen dioxide $\left(\mathrm{NO}_{2}\right)$, and ambient temperature for pregnant women with and without asthma. Methods: Women ( $n=40)$ from the Breathe-Well-Being, Environment, Lifestyle, and Lung Function Study (2015-2018) were enrolled during pregnancy and monitored for 2-4 days. Daily pollutants were measured using personal air monitors, indoor air monitors, and nearest Environmental Protection Agency's stationary monitors based on GPS tracking and home address. Results: Personal-monitor measurements of $\mathrm{PM}_{2.5}$, ozone, and $\mathrm{NO}_{2}$ did not vary by asthma status but exposure profiles significantly differed by assessment methods. EPA stationary monitor-based methods appeared to underestimate $\mathrm{PM}_{2.5}$ and temperature exposure and overestimate ozone and $\mathrm{NO}_{2}$ exposure. Higher indoor-monitored $\mathrm{PM}_{2.5}$ exposures were associated with smoking and the use of gas appliances. The proportion of waking-time during which personal monitors were worn was $\sim 56 \%$. Lower compliance was associated with exercise, smoking, being around a smoker, and the use of a prescription drug. Conclusions: Exposure did not vary by asthma status but was influenced by daily activities and assessment methods. Personal monitors may better capture exposures but non-compliance merits attention. Meanwhile, larger monitoring studies are warranted to further understand exposure profiles and the health effects of air pollution during pregnancy.

Keywords: air pollution; pregnancy; asthma; personal air monitoring; exposure assessment; monitoring compliance

\section{Introduction}

Asthma is characterized by inflammation of the airway, resulting in swelling that can adversely affect breathing. It is a common chronic disease affecting approximately $8 \%$ of pregnant women and is 
associated with pregnancy complications [1]. The course of asthma changes throughout pregnancy, with approximately one-third of the affected women having improved symptoms, one-third having worsened symptoms, and one-third having no change in symptoms, although women with more severe asthma at baseline have been shown to have a greater chance of exacerbation during pregnancy [2,3]. The causes of these changes are unclear but they may be influenced by infection, gastroesophageal reflux, changes in medication regimen, and smoking [2].

Ubiquitous environmental exposures, such as air pollution, increase the risk of asthma exacerbation in the general population through oxidative stress and inflammatory responses [4,5]. However, the effects of common air pollutants on asthma among pregnant women have not been well investigated. Asthma has been associated with higher risk for preeclampsia [6] and preterm birth [7] as well as neonatal respiratory complications associated with air pollutants but not all adverse outcomes of pregnancy vary by asthma status [8,9]. Regardless of asthma status, air pollution exposure appears to increase the risk for adverse pregnancy outcomes including pregnancy loss, stillbirth, preterm delivery and infant growth restriction [10-14]. Due to feasibility constraints, existing studies on the health effects of air pollution often rely on indirect estimates of air pollution exposure. For example, many earlier studies rely on measurements at the closest stationary air monitor to a location of interest such as a person's residential address [15]. This method is simple and easy to implement but is highly subject to exposure misclassification as it cannot adequately capture small spatial and temporal variability. In other words, it assumes that a person is exposed to the same levels of air pollution recorded at the closest stationary monitor to their home, which could be tens of miles away.

Complex mathematical models have also been developed to allow flexibility in incorporating environmental parameters (e.g., weather) that influence the spatiotemporal distribution of air pollution $[10,15]$. While more sophisticated models can provide more spatiotemporally accurate estimates, they are still indirect estimates, and are likely influenced by unverifiable model assumptions. Additionally, these models still treat exposure as a point source and cannot account for daily activity patterns. Global positioning system (GPS)-based technologies have also been used to improve estimation by capturing local mobility throughout the day [16].

Since people are mobile throughout the day, the best method for assessment of air pollution exposure is personal air monitoring, which requires participants to wear air monitors throughout the day. This method captures what a person breathes more accurately and can improve the ability to determine the true effects of air pollution on health. However, because personal monitoring is expensive and burdensome to the participants, it is difficult to implement. Since most of the population spend the majority of their time indoors, studies have also used indoor monitors to estimate personal exposure [17]. This method is thought to be more accurate than the indirect methods and can alleviate some of the daily burden of personal monitoring for study participants, thus improving compliance. Few studies have simultaneously implemented and compared these exposure assessment methods.

Pregnancy is a relatively short window of susceptibility, which presents a unique opportunity to assess air pollution exposures using personal monitoring together with other assessment methods. Whereas wearable monitors may improve the quantification of personal exposure, very few studies have simultaneously compared their feasibility with other common and less expensive assessment methods for pregnancy studies. The purpose of this study was to characterize air pollution exposures among pregnant women with and without asthma using four methods based on (1) the closest air monitor to residence, (2) the closest air monitor to GPS-monitored locations throughout the day, (3) indoor air monitors, and (4) personal portable air monitors. Furthermore, we also explored daily activities that can affect air pollution exposures and investigated factors that may influence the proportion of time participants actively wear the personal monitor during the monitoring period (i.e., personal monitoring compliance). 


\section{Methods}

\subsection{Study Sites}

Our study sites include the Center for Women's Reproductive Health at the University of Alabama, Birmingham, Alabama; and Northwestern University in Chicago, Illinois. These two sites have different environmental profiles and demographics. Chicago is a more metropolitan area and generally has higher air pollution concentrations. A comparison of the characteristics of these two areas can be found in Table S1.

\subsection{Data and Participants}

The Breathe-Well-Being, Environment, Lifestyle, and Lung Function Study (B-WELL-MOM) is a multi-center prospective cohort study (2015-2018) that aims to investigate factors associated with poor asthma control during pregnancy, as well as the basic immunology of asthma in pregnancy. Pregnant women were eligible for enrollment if they were $\geq 18$ years old; $<15$ weeks of gestation with a single gestation; an English or Spanish speaker; without a diagnosis of morbidities associated with immunologic alterations (e.g., HIV, multiple sclerosis, rheumatoid arthritis or mixed connective tissue disease); not expecting to terminate pregnancy; and planning to deliver at one of the study hospitals. To be classified in the active asthma study group, women with a history of asthma must have had symptoms or used prescription medication for asthma in the year prior to pregnancy. To be classified in the no asthma study group, women must have no history of an asthma diagnosis and no current asthma. The original cohort enrolled a total of 418 women including 147 with well-controlled asthma, 164 with poorly controlled asthma, and 107 with no asthma, defined by the American College of Obstetrics and Gynecology's classification of asthma severity during pregnancy based on medical records and self-reports [18]. Pregnant women completed a clinical interview with centrally trained interviewers, performed a $24 \mathrm{hr}$ dietary recall, and provided biological specimen during four study visits at gestational weeks $<15,20-22,30-32$, and at four months post-partum. At delivery, medical chart abstraction was used to obtain detailed clinical information. In addition, infant anthropometric measures were obtained at birth and at the post-partum visit. Women also completed daily diary and in-home assessments of respiratory functions (peak flow measurements and exhaled nitrous oxide) during pregnancy (Figure 1). The present analysis includes a subset of 40 pregnant women enrolled in the B-WELL-MOM study who agreed to participate in the air-monitoring sub-study.

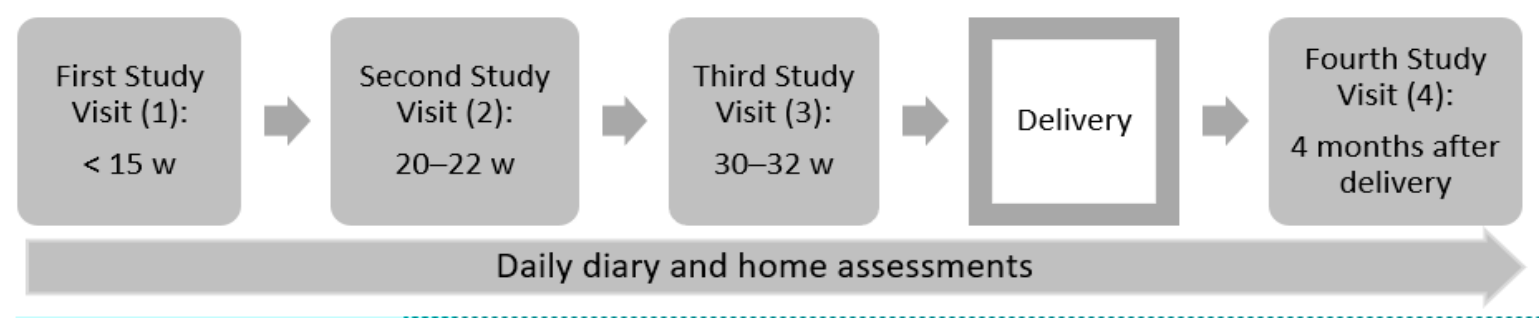

Figure 1. Schematic Timeline of B-WELL-MOM Study Visits.

\subsection{Air Pollution Assessment}

During study visits 2 or 3, pregnant women were asked to participate in the 4 day air-monitoring sub-study and were given wearable real-time sensors and passive badges to collect personal exposures and indoor air quality measurements. RTI MicroPEM ${ }^{\mathrm{TM}}$ wearable real-time sensors were used to collect $\mathrm{PM}_{2.5}$ data and filter samples; Cairpol CairClip (Altech) real-time sensors for $\mathrm{NO}_{2}$; and two Ogawa passive badges for $\mathrm{O}_{3}$ (Figure S1) [19]. These sensors have light weights (230, 55, and $18 \mathrm{~g}$, respectively) and compact sizes to be used as low-burden personal monitors for pregnant women. MicroPEM, CairClip and Ogawa badges were the best available technologies for wearable sensors when those real-time sensors were chosen in 2015 [20-22]. Prior to each MicroPEM deployment, each individual 
MicroPEM's flow rate and baseline response were calibrated [23]. The real-time $\mathrm{PM}_{2.5}$ concentration data were post-corrected using the corresponding gravimetrically determined concentration measured by the filter samples (gold-standard measurement). Real-time sensor-operating parameters such as inlet pressure drop, orifice pressure drop, and flow rate were examined during the validity check. The MicroPEM units were set to run for at least 5 days continuously using 30 second on/off cycling at a flow rate of $0.5 \mathrm{~L} / \mathrm{min}$. All CairClips were examined for precision through a collocation study before the air-monitoring study began. For Ogawa badges, shipping and lot blank samples were collected throughout the study to correct for potential contamination and background levels, respectively.

Participants were instructed to carry the monitors with them in a mesh pouch for 48 hours (personal assessment) and then place them in the main living quarter of their home (i.e., where people spend the majority of their time) for 48 hours (indoor assessment) (Figure S2). Real-time sensors (MicroPEM's, CairClips) were operated continuously throughout the monitoring period, and each of two Ogawa badges was deployed to collect separate personal and indoor samples. Participants also carried a tablet device for mobility tracking, and to complete a daily diary to assess daily activities as well as self-reported symptoms. Due to privacy concerns, we restricted the resolution of the mobility measure to 100 meters (approximately the length of one football field) so that exact locations were not collected. To ensure proper sensor deployment, utility and retrieval, video instructions were made available to both study staff and participants (Table S2). Informed consent was obtained from all participants and the study protocol was approved by Institutional Review Boards from all participating institutions.

In addition to personal and indoor monitoring, pollutants were also assessed using two common methods: stationary EPA monitor + home address, and stationary EPA monitor + GPS locations. First, participants' home addresses were spatiotemporally linked to the nearest EPA air monitor (average distance: $11.2 \mathrm{~km}$; range: $2.0-19.3 \mathrm{~km}$ ). Daily average concentrations of $\mathrm{PM}_{2.5}, \mathrm{NO}_{2}, \mathrm{O}_{3}$, and temperature for each woman were estimated during the 4 days of the air-monitoring sub-study using observed measurements at the nearest EPA monitor. Second, participants' GPS locations throughout the day (approximately every five minutes) during the monitoring period were spatiotemporally linked to the nearest EPA air monitor (average distance: $11.8 \mathrm{~km}$; range: 0.4 and $47.7 \mathrm{~km}$ ). Daily exposure to specific air pollutants during the study period was then estimated using the average location-specific concentrations observed at the nearest EPA monitors.

\subsection{Daily Activities}

During the monitoring period, pregnant women were asked to self-report daily activities through an app on their tablet, or on a written form. These activities include whether they spent time near heaters, burning trash, or smokers; used a gas range/oven/cooktop, water heater, gasoline equipment's, or printer.

\subsection{Monitor Compliance Assessment}

The MicroPEM device assesses participant activity level using an internal 3-axis accelerometer, which was used to validate participants' compliance of wearing monitors. Monitor compliance was operationalized as the proportion of time the monitor was worn during waking hours. This was calculated using the activity level data recorded by the accelerometer of the MicroPEM and self-reported sleeping hours, if provided. If sleeping hour information was not available, we assumed an $8 \mathrm{~h}$ sleep time per day.

\subsection{Predictors of Compliance}

Potential predictors of compliance were obtained using a combination of questionnaires, daily diaries, and in-home assessments. Maternal age, body mass index (BMI), and asthma status were assessed using questionnaires at baseline visit. Symptoms and daily activities were assessed using the daily diaries. Peak expiratory flow was collected in the morning and afternoon during daily in-home 
assessment using the Pocket Peak ${ }^{\circledR}$ Mechanical Peak Flow Meter (nSpire Health, Inc., Longmont, CO, USA). Exhaled nitrous oxide was collected during in-home assessment using the NIOX Vero (Aerocrine). Women were provided with proper training on how to use these devices during their clinical visits and were provided detailed instructions. In addition, daily diaries also assessed symptoms and various daily activities during the monitoring period.

\subsection{Statistical Analysis}

Fisher's exact test and t-test were used to compare time-invariant categorical and continuous characteristics between women with and without asthma, respectively. For time-varying factors (i.e., daily air pollution), mixed models were used to account for within-woman variations. To determine the types of activities (yes/no) associated with daily air pollution exposures, we used generalized linear mixed models. Since the distributions for pollutants concentrations were positively skewed, $\log$ transformation was used prior to modelling. The effect estimates were then converted to percent difference (and respective 95\% confidence intervals) in concentrations of air pollutants associated with specific activities. To determine factors associated with waking-hour monitor-wearing time (i.e., compliance), generalized linear mixed models were used to estimate the differences in monitoring wearing time associated with factors such as baseline asthma status, age, BMI, respiratory function measures, and various daily activities and symptoms. Given that the goal of our analysis was to explore predictors of monitor-wearing time, and the low sample size, we only used univariable models for our main analyses. Models with all significant factors yielded generally consistent conclusion with univariate models, so we opted to present the results of univariable models.

\section{Results}

The analysis includes a total of 40 pregnant women who participated in the air pollution-monitoring sub-study (with 24 provided GPS data). There were 19 (47.5\%) women with well-controlled asthma, $12(30.0 \%)$ with poorly controlled asthma, and $9(22.5 \%)$ without asthma at baseline (Table 1$)$. Women with asthma generally enrolled in this sub-study earlier in pregnancy compared to their counterparts (gestational week 23 vs. $27, p=0.06)$. The majority of pregnant women $(72.5 \%)$ were $\geq 30$ years old or overweight/obese $(80.0 \%)$. Women actively wore the air monitors for approximately $55.7 \%$ of the monitoring time during waking hours and reported spending an average of 93 minutes outdoors per day. These characteristics did not differ by baseline asthma status (Table 1).

Average home distance from EPA air monitors ranged from 6.3 to $23.5 \mathrm{~km}$ depending on the types of pollutant, and was independent of baseline asthma status. Temperature monitors were typically further from participants' home (Table 1), as temperature monitoring is not always available at all monitoring stations. GPS distance from the nearest EPA air monitor did vary by asthma status. Women with poorly controlled asthma tended to be closer to $\mathrm{PM}_{2.5}$ monitors $(5.8 \mathrm{~km}$ vs. 8.1 and $6.3 \mathrm{~km}$, $p<0.01$ ), ozone monitors $(7.2 \mathrm{~km}$ vs 9.3 and $8.1, p<0.01)$ and $\mathrm{NO}_{2}$ monitors $(4.3 \mathrm{~km}$ vs. 7.0 and 8.1 , $p<0.01$ ) compared to women with well-controlled asthma or no asthma (Table 1). This finding is consistent when excluding the one GPS participant from one of the two sites (not shown). Women with poorly controlled asthma had lower peak flow values, and higher rates of wheezing.

Overall, $\mathrm{PM}_{2.5}$, ozone, $\mathrm{NO}_{2}$, and temperature exposures among asthmatic and non-asthmatic pregnant women did not differ significantly when estimated with personal or indoor monitors (Table 2, Figure S3). However, women with asthma tend to be exposed to higher $\mathrm{O}_{3}$ concentrations and higher ambient temperature compared to their counterparts when estimated using EPA monitors measurements. More specifically, when exposures were estimated using GPS or home address locations + the EPA monitor method, women with poorly controlled asthma were exposed to a daily $\mathrm{O}_{3}$ average of $33.9 \mathrm{ppb}$ compared to $28.4 \mathrm{ppb}$ among well-controlled and $24.6 \mathrm{ppb}$ among non-asthmatics $(p=0.01)$. Similarly, women with poorly controlled asthma were exposed to higher ambient temperature $\left(15.9^{\circ} \mathrm{C}\right.$ vs. 12.1 and $9.8^{\circ} \mathrm{C}, p=0.01$ ) compared to their counterparts (Table 2, Figure S3). These results are consistent even when excluding the one participant from one of the sites (Table S3). 
Table 1. Characteristics of B-WELL-MOM participants who participated in the air-monitoring sub-study $(n=40)$.

\begin{tabular}{|c|c|c|c|c|c|}
\hline \multirow[b]{2}{*}{ Characteristics } & \multicolumn{5}{|c|}{$n(\%)$ or Mean $(\mathrm{SD})$} \\
\hline & $\begin{array}{l}\text { Overall } \\
(n=40)\end{array}$ & $\begin{array}{l}\text { No Asthma } \\
(n=9,22.5 \%)\end{array}$ & $\begin{array}{l}\text { Well Controlled } \\
(n=19,47.5 \%)\end{array}$ & $\begin{array}{l}\text { Poorly Controlled } \\
\quad(n=12,30.0 \%)\end{array}$ & $p^{\mathrm{a}}$ \\
\hline \multicolumn{6}{|l|}{ Study site (n, \%) } \\
\hline Site 1 & $36(90.0)$ & $8(88.9)$ & $18(94.7)$ & $10(83.3)$ & 0.67 \\
\hline Site 2 & $4(10.0)$ & $1(11.1)$ & $1(5.3)$ & $2(16.7)$ & \\
\hline Gestational age at enrollment (week, mean, SD) & $24.0(4.6)$ & $27.1(5.5)$ & $23.1(4.5)$ & $23.1(2.8)$ & 0.06 \\
\hline \multicolumn{6}{|l|}{ Maternal age group (year, n, \%) } \\
\hline$<23$ & $1(2.5)$ & $1(11.1)$ & $0(0)$ & $0(0)$ & 0.42 \\
\hline $23-29$ & $10(25.0)$ & $1(11.1)$ & $5(26.3)$ & $4(33.3)$ & \\
\hline$\geq 30$ & $29(72.5)$ & $7(77.8)$ & $14(73.7)$ & $8(66.7)$ & \\
\hline \multicolumn{6}{|l|}{ Maternal body mass index $\left(\mathrm{kg} / \mathrm{m}^{2}, \mathrm{n}, \%\right)$} \\
\hline Normal & $8(20.0)$ & $1(11.1)$ & $5(26.3)$ & $2(16.7)$ & 0.68 \\
\hline Overweight/obese & $32(80.0)$ & $8(88.9)$ & $14(73.7)$ & $10(83.3)$ & \\
\hline Monitor-wearing time (\%, mean, range) & $38.4(16.5-85.2)$ & $39.2(16.6-69.2)$ & $40.4(16.5-77.1)$ & $44.9(24.3-85.2)$ & 0.73 \\
\hline Waking-hour monitor-wearing time (\%, mean, range) & $55.7(22.8-100.0)$ & $59.6(22.8-100)$ & $53.9(24-100)$ & $55.5(30.8-100)$ & 0.85 \\
\hline \multicolumn{6}{|l|}{ GPS monitoring } \\
\hline Yes & $24(60.0)$ & $5(55.6)$ & $13(68.2)$ & $6(50.0)$ & 0.64 \\
\hline No & $16(40.0)$ & $4(44.4)$ & $6(31.6)$ & $6(50.0)$ & \\
\hline Reported time spent outdoors per day (mins, mean, range) & $93.3(0-480)$ & $94.2(0-360)$ & $98.6(0-480)$ & $83.1(0-300)$ & 0.67 \\
\hline \multicolumn{6}{|c|}{ Average home distance from nearest EPA monitor (km, mean, range) } \\
\hline $\mathrm{PM}_{2.5}$ & $6.6(0.67-14.0)$ & $6.2(4.1-8.6)$ & $6.8(0.7-14)$ & $6.7(4.2-9.5)$ & 0.97 \\
\hline Temperature & $23.5(4.1-34.7)$ & $18.7(4.1-29.2)$ & 23.1(11.2-29.3) & 28.2(21.4-34.7) & 0.09 \\
\hline Ozone & $8.5(2.3-13.6)$ & $8.6(5.3-11.8)$ & $8.7(2.3-14.6)$ & $8.2(4.2-11.9)$ & 0.96 \\
\hline $\mathrm{NO}_{2}$ & $6.3(0.75-14.0)$ & $6.6(0.8-13.4)$ & $7.1(1.0-14.0)$ & $4.3(1.8-8.3)$ & 0.38 \\
\hline \multicolumn{6}{|c|}{ Average GPS distance from nearest EPA monitor ( $\mathrm{km}$, mean, range) } \\
\hline $\mathrm{PM}_{2.5}$ & $6.9(0.38-43.8)$ & $6.3(0.4-17.1)$ & $8.1(0.5-43.8)$ & $5.8(0.7-9.5)$ & $<0.01$ \\
\hline Temperature & $25.6(0.38-63.5)$ & $19.1(0.4-63.5)$ & $24.7(2.1-30.9)$ & $30.8(12.6-42.3)$ & $<0.01$ \\
\hline Ozone & $8.3(0.38-25.2)$ & $8.1(0.4-25.2)$ & $9.3(0.8-25.1)$ & $7.2(1.4-13.9)$ & $<0.01$ \\
\hline $\mathrm{NO}_{2}$ & $6.3(0.36-58.2)$ & $8.1(0.4-58.2)$ & $7(0.6-55.9)$ & $4.3(0.7-13.9)$ & $<0.01$ \\
\hline
\end{tabular}


Table 1. Cont

\begin{tabular}{|c|c|c|c|c|c|}
\hline \multirow[b]{2}{*}{ Characteristics } & \multicolumn{5}{|c|}{$n(\%)$ or Mean $(\mathrm{SD})$} \\
\hline & $\begin{array}{l}\text { Overall } \\
(n=40)\end{array}$ & $\begin{array}{c}\text { No Asthma } \\
(n=9,22.5 \%)\end{array}$ & $\begin{array}{c}\text { Well Controlled } \\
(n=19,47.5 \%)\end{array}$ & $\begin{array}{l}\text { Poorly Controlled } \\
\quad(n=12,30.0 \%)\end{array}$ & $p^{\mathrm{a}}$ \\
\hline Exhaled nitric oxide (ppb, mean, range) & $17.6(5-52)$ & $21.5(5-44)$ & $20(7-48)$ & $15.1(5-52)$ & 0.29 \\
\hline Max peak flow morning (L/min, mean, range) & $377.3(130-650)$ & $406.3(150-650)$ & $418(260-540)$ & $358(160-550)$ & $<0.01$ \\
\hline Max peak flow afternoon (L/min, mean, range) & $340.4(100-540)$ & $384.8(140-540)$ & $379.4(210-500)$ & $323.2(100-510)$ & $<0.01$ \\
\hline \multicolumn{6}{|c|}{ Rate of events (per 100 person-day reported, mean, range) } \\
\hline Wheeze & $7.3(0-100)$ & $0(0-0)$ & $3.1(0-33.3)$ & $19.6(0-100)$ & $<0.01$ \\
\hline Cough & $27.2(0-100)$ & $30.6(0-100)$ & $27.9(0-100)$ & $23.4(0-75.0)$ & 0.10 \\
\hline Shortness of breath & $31.5(0-100)$ & $28.7(0-100)$ & $26.9(0-100)$ & $41.0(0-100)$ & 0.20 \\
\hline Chest tightness & $7.1(0-100)$ & $4.0(0-25.0)$ & $10.8(0-100)$ & $3.7(0-25)$ & 0.09 \\
\hline Chest pain & $2(0-25)$ & $5.0(0-25.0)$ & $1.1(0-20.0)$ & $1.2(0-14.3)$ & 0.38 \\
\hline Nausea & $17(0-100)$ & $20.8(0-75.0)$ & $12.8(0-80.0)$ & $20.7(0-100)$ & 0.65 \\
\hline Runny nose & $47.5(0-100)$ & $33.3(0-100)$ & $56.1(0-100)$ & $44.5(0-100)$ & $<0.01$ \\
\hline Missed work & $3.5(0-60)$ & $2.8(0-25.0)$ & $5.3(0-60.0)$ & $1.2(0-14.3)$ & 0.45 \\
\hline Wake up at night & $14.8(0-100)$ & $33.6(0-100)$ & $4.2(0-80.0)$ & $17.7(0-100)$ & 0.07 \\
\hline Exercise & $21.1(0-100)$ & $12.9(0-40.0)$ & $22.5(0-100)$ & $25.1(0-100)$ & 0.49 \\
\hline Smoke & $2.5(0-100)$ & $0(0-0)$ & $0(0-0)$ & $8.3(0-100)$ & 0.31 \\
\hline Around a smoker & $10(0-100)$ & $16.1(0-100)$ & $5.4(0-66.7 .0)$ & $12.8(0-100)$ & 0.36 \\
\hline
\end{tabular}

across asthma status and were obtained from exact tests for categorical variables, and mixed linear models for continuous variables to account for within-woman variation.
a 
Pollutant estimates were significantly different across different assessment methods (Table 2, Figure S3). Compared to EPA monitor-based assessment methods, personal and indoor air monitors generally captured higher concentrations of $\mathrm{PM}_{2.5}$ exposures and higher ambient temperature, but lower concentrations of $\mathrm{O}_{3}$ and $\mathrm{NO}_{2}$, both of which are generally outdoors pollutants. It is also important to notice that personal and indoor monitors captured more variability, including $\mathrm{PM}_{2.5}$ concentrations that reached well above the EPA's daily standard of $35 \mathrm{ug} / \mathrm{m}^{3}$ (Table 2, Figure S3) [24]. The findings are generally consistent for when stratified by study sites (Table S4). A correlation matrix with air pollutant exposures estimated by different assessment methods is also presented in Table S5. In general, there is strong correlation between GPS- and home-based estimation methods for all pollutants. Personally measured exposures to $\mathrm{PM}_{2.5}(\mathrm{r}=0.32), \mathrm{NO}_{2}(\mathrm{r}=0.76)$, and temperature $(\mathrm{r}=0.76)$ were positively associated with indoor-monitor measurements. Estimates using EPA monitors were generally not positively correlated with personal or indoor estimates, except for temperature.

Table 3 describes the percent difference in exposures associated with various daily activities. Pregnant women who used gas appliances during monitoring time (e.g., cook top or oven) had 194.6\% (95\% CI: 55.0-549.6\%) higher indoor exposures to $\mathrm{PM}_{2.5}$ compared to those who did not. Those who smoked also had almost 400\% (95\% CI: 78.5-1293.8\%) higher indoor concentrations of $\mathrm{PM}_{2.5}$. Generally, using a gas appliance, clothes dryer, or a printer and spending time near a heater, burning trash, water heater, or smoking were associated with higher exposures to $\mathrm{PM}_{2.5}, \mathrm{O}_{3}$, and $\mathrm{NO}_{2}$, but these associations were not statistically significant, possibly due to the low sample size.

Compliance for women in the study, defined by the percent of time air monitor was worn during waking time, was approximately $55.7 \%$, and did not differ by asthma status (Table 4). Having a missed workday and being near a heater was associated with approximately a 20\% (95\% CI: $1-39 \%)$ and $42 \%$ (34-50\%) increase in compliance, respectively. On the other hand, exercise (-11\%, 95\%CI: $-19--3 \%)$, smoking $(-23 \%, 95 \% \mathrm{CI}:-30--16 \%)$, being around a smoker $(-16 \%, 95 \% \mathrm{CI}:-26--6 \%)$, and using a prescription drug $(-13 \%, 95 \%$ CI: $-26--0.4 \%)$ were associated with significantly lower waking-hour monitor-wearing time. When all significant factors were included in a single model, results were consistent, except missing a work day was no longer significant (data not shown). 
Table 2. Distribution of air pollution exposure by asthma status.

\begin{tabular}{|c|c|c|c|c|c|c|}
\hline \multirow[t]{2}{*}{ Pollutant } & \multirow[t]{2}{*}{ Assessment Method a } & \multicolumn{5}{|c|}{ Mean (Min-Max) } \\
\hline & & All & No Asthma & Well Controlled & Poorly Controlled & $p$-Value ${ }^{b}$ \\
\hline \multirow[t]{5}{*}{$\mathrm{PM}_{2.5}\left(\mu \mathrm{g} / \mathrm{m}^{3}\right)$} & Home + EPA monitor & $9.1(1.7-28.5)$ & $9.7(3.6-19)$ & $8.5(1.7-16)$ & $9.2(3.6-28.5)$ & 0.35 \\
\hline & GPS + EPA monitor & $9.3(1.7-28.5)$ & $10.3(3.6-28)$ & $8.4(1.7-14.6)$ & $9.2(3.6-28.5)$ & 0.11 \\
\hline & Indoor & $14.2(1-132.8)$ & $12.5(2.3-76.5)$ & $15.8(1-132.8)$ & $12.8(2.5-85.8)$ & 0.69 \\
\hline & Personal & $26.7(0.9-665.6)$ & $15.1(2.1-122.3)$ & $42.2(0.9-665.6)$ & $12.1(2.4-44.7)$ & 0.17 \\
\hline & $p$-value exposure ${ }^{c}$ & 0.001 & 0.42 & 0.01 & 0.22 & \\
\hline \multirow[t]{4}{*}{ Ozone (ppb) } & Home + EPA monitor & $28.9(5.3-68.8)$ & $24.6(5.3-65)$ & $28.4(5.4-59.5)$ & $33.9(6.3-68.8)$ & 0.01 \\
\hline & Indoor & $2.6(1.1-23.9)$ & $2.2(1.6-3)$ & $3.2(1.1-23.9)$ & $2.1(1.1-3.2)$ & 0.16 \\
\hline & Personal & $3.1(1.5-12.2)$ & $3.5(1.9-7.6)$ & $2.7(1.9-5.5)$ & $3.4(1.5-12.2)$ & 0.13 \\
\hline & $p$-value exposure ${ }^{c}$ & $<.0001$ & $<0.0001$ & $<0.0001$ & $<0.0001$ & \\
\hline \multirow[t]{5}{*}{$\mathrm{NO}_{2}(\mathrm{ppb})$} & Home + EPA monitor & $16.3(4-35.8)$ & $17.3(6.9-31)$ & $15.7(5.9-35.8)$ & $16.5(4-35)$ & 0.42 \\
\hline & GPS + EPA monitor & $16.5(4-35)$ & $18.3(7.9-31.6)$ & $15.6(5.5-34.7)$ & $16.4(4-35)$ & 0.07 \\
\hline & Indoor & $4.6(0-39)$ & $3.5(0-15.8)$ & $5.1(0-27.9)$ & $4.7(0-39)$ & 0.66 \\
\hline & Personal & $5.1(0-27.4)$ & $4.8(0-17.4)$ & $5.6(0-27.4)$ & $4.5(0-23.1)$ & 0.67 \\
\hline & $p$-value exposure ${ }^{c}$ & $<0.0001$ & $<0.0001$ & $<0.0001$ & $<0.001$ & \\
\hline \multirow{4}{*}{ Temperature $\left({ }^{\circ} \mathrm{C}\right)$} & GPS + EPA monitor & $12.6(-16.3-28.8)$ & $9.7(-4.4-27.8)$ & $12(-16.3-28.8)$ & $15.9(-4.8-27)$ & 0.01 \\
\hline & Indoor & $24.3(17.1-52.8)$ & $25(19.5-31.7)$ & $24.4(17.1-52.8)$ & $23.7(18.1-31)$ & 0.38 \\
\hline & Personal & $23.6(13.1-38.7)$ & $24.5(13.1-38.7)$ & $23.6(16.8-33.7)$ & $23(16-30.3)$ & 0.36 \\
\hline & $p$-value exposure ${ }^{c}$ & $<0.0001$ & $<0.0001$ & $<0.001$ & $<0.001$ & \\
\hline
\end{tabular}

GPS, global positioning system; EPA, US Environmental Protection Agency; $\mathrm{PM}_{2.5}$, particulate matter <2.5 microns; $\mathrm{NO}_{2}$, nitrogen dioxide; ${ }^{a}$ The GPS and home method includes status by mixed models to account for within-person variation. ${ }^{c} p$-values were obtained for comparison across assessment methods by mixed models to account for within-person variation. 
Table 3. Percent difference (and 95\% confidence intervals) in air pollutants associated with specific daily activities.

\begin{tabular}{|c|c|c|c|c|c|c|}
\hline \multirow{2}{*}{$\begin{array}{l}\text { Activities (Yes vs. } \\
\text { No) }\end{array}$} & \multicolumn{2}{|c|}{$\mathrm{PM}_{2.5}\left(\mu \mathrm{g} / \mathrm{m}^{3}\right)$} & \multicolumn{2}{|c|}{$\mathrm{NO}_{2}(\mathrm{ppb})$} & \multicolumn{2}{|c|}{ Ozone (ppb) } \\
\hline & Personal & Indoors & Personal & Indoors & Personal & Indoors \\
\hline Near heater & $-43.0(-88.7,187.6)$ & - & $170.9(-91.0,8040.1)$ & - & $20.7(-33.6,119.2)$ & - \\
\hline Near burning trash & $28.8(-69.0,435.1)$ & $68.3(-54.7,524.8)$ & $28.8(-91.4,1831.2)$ & $276.6(-91.2,16,070.4)$ & $-8.2(-45.7,55.1)$ & $11.7(-40.5,109.8)$ \\
\hline Used gas appliance & $46.4(-21.3,172.5)$ & $194.6(55.0,459.6) *$ & $33.2(-53.5,281.4)$ & $92.4(-72.9,1268.3)$ & $-17.1(-34,4.0)$ & $-3.1(-35.5,45.7)$ \\
\hline Used clothes dryer & $11.6(-48.1,140.0)$ & $126.1(-20.3,541.4)$ & $58.2(-70.6,752.3)$ & $31.6(-92.3,2133.9)$ & $0.7(-24.5,34.3)$ & $10.9(-34.8,88.5)$ \\
\hline Near water heater & $-11.3(-99.4,12,893.3)$ & $68.4(-61.5,636.1)$ & $52.1(-99.9,209,422.1)$ & $549.8(-60.6,10,626.8)$ & $51.4(-50.3,361.0)$ & $40.8(-25.5,166.2)$ \\
\hline Used printer & $4.0(-46.3,101.5)$ & - & $-26.9(-77.1,133.7)$ & - & $-19.4(-36.5,2.3)$ & - \\
\hline Smoking & $88.2(-83.5,2048)$ & $398.7(78.5,1293.8) *$ & $66.9(-95.8,6499.9)$ & $\begin{array}{c}1008.9(-67.5 \\
37,779.1)\end{array}$ & $47.4(-5.5,130.0)$ & $12.3(-40.2,110.8)$ \\
\hline Near smoker & $29.9(-87.8,1283.9)$ & $3.1(-67.7,229.1)$ & $-78.0(-99.5,844.9)$ & $18.5(-99.3,20,321.0)$ & $54.1(-15.4,180.7)$ & $-0.2(-33.7,50.1)$ \\
\hline Exercise & $-1.6(-47.1,83.2)$ & $36.3(-25.8,150.3)$ & $-0.6(-56.2,125.5)$ & $-19.2(-78.5,203.1)$ & $1.8(-13.8,20.1)$ & $8.7(-12.2,34.6)$ \\
\hline
\end{tabular}

$\mathrm{PM}_{2.5}$, particulate matter $<2.5$ microns; $\mathrm{NO}_{2}$, nitrogen dioxide; Blank cell indicates that the model did not converge due to small numbers; Linear mixed models adjusted for within-person variation; air pollutants were log transformed; * Indicates statistical significance at alpha $<0.05$. 
Table 4. Factors associated with compliance (proportion of waking-hour monitor-wearing time).

\begin{tabular}{|c|c|c|}
\hline Characteristics & $\beta(95 \% \mathrm{CI})$ & $p^{a}$ \\
\hline \multicolumn{3}{|l|}{ Asthma status } \\
\hline No asthma & reference & \\
\hline Well controlled & $-4.23(-23.1,14.64)$ & 0.66 \\
\hline Poorly controlled & $-4.68(-26.15,16.78)$ & 0.67 \\
\hline \multicolumn{3}{|l|}{ Site } \\
\hline Site 1 & reference & \\
\hline Site 2 & $11.73(-8.08,31.54)$ & 0.25 \\
\hline \multicolumn{3}{|l|}{ Maternal age group (years) } \\
\hline$<23$ & reference & \\
\hline $23-29$ & $7.60(-7.93,23.12)$ & 0.34 \\
\hline$\geq 30$ & $7.48(-1.42,16.38)$ & 0.10 \\
\hline \multicolumn{3}{|l|}{ Maternal body mass index } \\
\hline Normal & reference & \\
\hline Overweight/obese & $-9.6(-26.77,7.57)$ & 0.27 \\
\hline Exhaled nitric oxide (ppb) & $0.1(-0.48,0.68)$ & 0.74 \\
\hline Max morning peak flow (L/min) & $-0.01(-0.06,0.04)$ & 0.66 \\
\hline Max afternoon peak flow (L/min) & $-0.02(-0.07,0.03)$ & 0.45 \\
\hline \multicolumn{3}{|l|}{ Symptoms (yes vs. no) } \\
\hline Wheeze & $-5.66(-14.94,3.62)$ & 0.23 \\
\hline Cough & $0.79(-13.34,14.93)$ & 0.91 \\
\hline Shortness of breath & $-2.99(-14.16,8.18)$ & 0.60 \\
\hline Chest tightness & $9.88(-10.92,30.67)$ & 0.35 \\
\hline Chest pain & $9.29(-6.76,25.34)$ & 0.26 \\
\hline Nausea & $13.27(-2,28.54)$ & 0.09 \\
\hline Runny nose & $2.06(-9.57,13.7)$ & 0.73 \\
\hline Missed work & $19.81(0.91,38.72)$ & 0.04 \\
\hline Wake up at night & $3.17(-15.87,22.2)$ & 0.74 \\
\hline Exercise & $-10.64(-18.66,-2.61)$ & 0.01 \\
\hline Smoke & $-22.83(-29.94,-15.71)$ & $<0.001$ \\
\hline Around a smoker & $-16.24(-26.43,-6.04)$ & $<0.001$ \\
\hline Used prescription drug & $-13.39(-26.42,-0.37)$ & 0.04 \\
\hline Used over the counter drug & $-10.37(-26.39,5.66)$ & 0.20 \\
\hline Near heater & $42.05(33.8,50.31)$ & $<0.001$ \\
\hline Near burning trash & $-7.33(-22.81,8.16)$ & 0.35 \\
\hline Used gas appliance & $-8.74(-22.99,5.5)$ & 0.23 \\
\hline Used clothes dryer & $-6.29(-20.58,8)$ & 0.39 \\
\hline Near water heater & $15.41(-6.13,36.95)$ & 0.16 \\
\hline Used printer & $-1.11(-15.94,13.72)$ & 0.88 \\
\hline Times spent outdoors (minutes) & $0.03(-0.04,0.1)$ & 0.38 \\
\hline
\end{tabular}

\section{Discussion}

The purpose of this study was to characterize air pollution exposures among pregnant women with and without asthma using four different assessment methods, including personal and indoor sensor measurements and estimation using home address and GPS location in combination with EPA ambient monitoring station data. We also explored daily activities that may affect air pollution exposures, and factors influencing the proportion of waking time during which monitors were worn.

Our analyses suggest that when measured with personal or indoor monitors, exposures to $\mathrm{PM}_{2.5}$, $\mathrm{O}_{3}, \mathrm{NO}_{2}$, and temperature did not differ by baseline asthma status. On the contrary, when estimated by EPA monitors (both based on home address and GPS movements), women with poorly controlled asthma appeared to have significantly higher exposures to $\mathrm{O}_{3}$ and higher ambient temperature 
independent of site differences. This may suggest that women with poorly controlled asthma may live and move around in neighborhoods with higher outdoor concentrations of $\mathrm{O}_{3}$ and warmer climates, both of which are not captured by personal monitors given that women spent most of their time indoors. Other studies examining outdoor air pollution and neighborhood environment have also shown that people living in more polluted neighborhoods may have worse asthma morbidity [25-28]. However, these differences have not been well investigated among pregnant women and merit further attention. Studies have also shown that vulnerable subgroups of the population often live and work in areas with warmer outdoor microclimate due to socioeconomic differences including greenspace and asphalt surfaces $[29,30]$. It is important to note that we examined exposures based on baseline asthma status, while it is possible that women's asthma status may change throughout pregnancy [31]. Thus, future studies should examine exposures in relation to asthma status change throughout pregnancy.

Our data also show that exposures varied significantly by assessment methods. More specifically, local outdoor monitor-based (e.g., EPA monitor) methods appeared to underestimate $\mathrm{PM}_{2.5}$ and ambient temperature, and overestimate $\mathrm{O}_{3}$ and $\mathrm{NO}_{2}$ exposures relative to personal and indoor monitors. The indoor-outdoor differences in $\mathrm{PM}_{2.5}$ and $\mathrm{O}_{3}$ are generally consistent with other studies in the US and around the world [32-34]. These differences have important implications for future studies, as they support the notion that local air monitors lack the ability to capture small spatial variations in different exposure microenvironments. Outdoor and indoor monitors also capture different sources of pollution but, since people spend most of their times indoor, indoor monitoring may have the greatest contribution to personal exposure, if compliance is reasonable. Our data also show that the GPS-based and home-based methods yielded similar estimates with high correlation, suggesting that accounting for outdoor space-time activities do not significantly influence exposure estimates [35].

Personal air monitors have the advantage of being able to capture more exposure variability, as shown in our data as well as other studies [36]. However, our data also suggest that compliance is approximately $56 \%$ of waking time, which presents an important challenge in environmental studies that involve personal monitoring. We also note that a prior study investigating personal exposure protocol compliance shows that although compliance can vary between people, it does not appear to misrepresent exposures measured longitudinally when one can reach a threshold of approximately $40 \%$ [37]. Given that women spend most of their time indoors [38], a trend that is also reflected in our data, indoor monitoring may offer a reasonable alternative to alleviate participant burden, especially considering our results which suggest that indoor estimates are very similar to measurements from personal monitors. We also observed that having a missed workday and being near a heater are associated with higher compliance, and this may suggest that women are more likely to wear the personal monitors when they are indoors.

Daily activities may influence exposures. Women who smoked and used gas appliances had significantly higher exposures to indoor fine particles, but they were also significantly less likely to wear monitors during waking hours. The differences in exposures based on these daily activities were reflected in indoor monitoring, which provides another reason indoor monitoring may be a reasonable alternative in future studies. In addition, some common daily activities, including the use of gas appliances, are associated with higher exposure to pollutants. As such, it is important to raise awareness regarding the potentially harmful effects of these pollutants and encourage strategies for minimizing exposure such as the use of vent hoods, air purifiers, and the avoidance of active/passive smoking. Meanwhile, there is a need for more comprehensive and larger studies to investigate personal exposure data together with daily diaries to identify activities that cause high exposure conditions in order to prepare effective and reasonable intervention efforts.

A study among physicians in Poland showed that only approximately one-quarter of physicians believed that their knowledge about the health effects of air pollution is sufficient, and only $3.5 \%$ answered the general knowledge questions correctly [39]. In the US, despite the well-described health effects of air pollution, the majority of physicians reported never talking to their patients about 
limiting exposures [40]. This suggests a clear need for tools and resources to help health care providers communicate the deleterious effects of air pollution [41], especially to vulnerable populations such as pregnant women. Meanwhile, well-designed resources for the general public are also necessary. These efforts need to ensure that the communication strategies include critical information including risk mitigation behaviors and health impacts, and that they reach vulnerable populations [42].

This study has a few limitations. First, daily activities and some symptoms are self-reported and depend on participants' ability to recall. To minimize this issue, participants were given a computer tablet accompanied by a user-friendly app to report daily symptoms. Since participants were not entirely aware of their air pollution exposures and that this study is prospective in nature, recall bias is not expected to be significant. There may also be concerns that the daily activities during the monitoring period may not reflect the normal daily activities. A cohort study has shown that $71 \%$ of participants reported that their activity while wearing the monitor is representative of normal activity [43]. Our study is also limited by the low sample size, especially for the GPS monitoring portion, leading to limited generalizability and greater chance of type II errors. In addition, emerging real-time sensors used in this study do not have the same data quality of the EPA reference monitors although participants mostly spent their time in indoor environments where the sensors perform better than in outdoor environments. We also note that due to the small sample size, we were not able to sufficiently estimate exposure by modelling as a method of assessment, nor perform sensitivity analysis excluding those with compliance below certain thresholds. Lastly, the proportion of waking-time during which personal monitors were worn was at most moderate, which potentially limits the generalizability and validity of personal monitoring. Nevertheless, this is the first study to compare air pollution exposures using different assessment strategies including personal monitoring among women with and without asthma. Our findings add to the limited literature on air pollution exposures during pregnancy and highlight the need for larger studies to understand air pollution exposures and their effects during pregnancy. Given the adverse outcomes of pregnancy previously shown to be associated with ambient air pollution, including pregnancy loss [14], stillbirth [11], preterm birth, and neonatal complications $[6,7,10,12,13]$, improving exposure assessment is a key component to strategies for improving health outcomes for this vulnerable population.

\section{Conclusions}

Pregnant women with and without asthma appeared to be exposed to the same level of air pollution when measured by personal or indoor air monitors. However, exposure profiles differed significantly by assessment method. Local outdoor monitor methods appeared to underestimate $\mathrm{PM}_{2.5}$ and ambient temperature, and overestimate $\mathrm{O}_{3}$ and $\mathrm{NO}_{2}$ exposures relative to personal and indoor monitors. Personal monitors worn throughout the day may best reflect true exposure and are feasible but compliance with continual use was not high, making indoor monitors a potentially more feasible alternative. Furthermore, given that pregnant women who reported certain daily activities (e.g., smoking and using a gas range) had higher air pollution exposures compared to those who did not, it is important to raise awareness on the health effects of air pollution, and strategies that can minimize exposures for pregnant women. These may include using vent hoods during cooking, taking advantage of air purifiers when necessary, and avoiding active and passive smoking. Improving exposure assessment will also allow us to better understand the effects of air pollution during pregnancy. Effective strategies to minimize air pollution exposures among pregnant women are warranted and may facilitate physician involvement in clinical translation to mitigate these potential risks for adverse outcomes among pregnant women with and without asthma.

Supplementary Materials: The following are available online at http://www.mdpi.com/1660-4601/17/13/4888/s1, Figure S1: Wearable sensors used in the study: RTI MicroPEM (left), Cairpol CairClip (middle), and $\mathrm{Ogawa} \mathrm{O}_{3}$ passive sampler (right); Figure S2: The air monitoring sensors in the monitoring platform worn by a model pregnant mother (left), mesh pouch including all monitors (middle), and shipping tray (right); Figure S3: Distribution of air pollution exposure by asthma status; Table S1: Selected characteristics of study areas; Table S2: Training video clips that include step-by-step instructions for personal air monitoring; Table S3: Air pollution distributions by 
asthma status for Site 1; Table S4: Air pollution distribution by study site; Table S5: Correlation matrix for different pollutants across assessment methods.

Author Contributions: Conceptualization, S.H. and P.M.; Methodology, all; Software, S.H.C., S.H., S.S., and J.K.; Formal Analysis, S.H. and J.K.; Data Curation, S.H.C., S.H., and S.S.; Writing-Original Draft, S.H.; Writing-Review and Editing, S.H., C.N., J.K., S.S., S.-H.C., N.P., A.W., W.G., J.B., A.S., M.O., Z.C. and P.M. All authors have read and agreed to the published version of the manuscript.

Funding: This study was supported by the Intramural Research Program of the Division of Intramural Population Health Research, Eunice Kennedy Shriver National Institute of Child Health and Human Development (contracts HHSN275201300013C, HHSN275201300014C, and HHSN275201300026I; task order no. HHSN27500001 and HHSN275000017), and the University of California Start-Up Funds. Although NICHD cleared the manuscript for publication, the funders had no role in the design and conduct of the study. All conclusions are that of the authors and do not necessarily represent the views of the funders.

Acknowledgments: The authors also wish to thank the participants and staff involved in the study.

Conflicts of Interest: The authors declare no conflict of interest.

\section{References}

1. Kelly, W.; Massoumi, A.; Lazarus, A. Asthma in pregnancy: Physiology, diagnosis, and management. Postgrad Med. 2015, 127, 349-358. [CrossRef] [PubMed]

2. Gluck, J.C.; Gluck, P.A. The effect of pregnancy on the course of asthma. Immunol. Allergy Clin. North. Am. 2006, 26, 63-80. [CrossRef]

3. Schatz, M.; Dombrowski, M.P.; Wise, R.; Thom, E.A.; Landon, M.; Mabie, W.; Newman, R.B.; Hauth, J.C.; Lindheimer, M.; Caritis, S.N.; et al. Asthma morbidity during pregnancy can be predicted by severity classification. J. Allergy Clin. Immunol. 2003, 112, 283-288. [CrossRef] [PubMed]

4. Guarnieri, M.; Balmes, J.R. Outdoor air pollution and asthma. Lancet 2014, 383, 1581-1592. [CrossRef]

5. Orellano, P.; Quaranta, N.; Reynoso, J.; Balbi, B.; Vasquez, J. Effect of outdoor air pollution on asthma exacerbations in children and adults: Systematic review and multilevel meta-analysis. PLoS ONE 2017, 12, e0174050. [CrossRef]

6. Mendola, P.; Wallace, M.; Liu, D.; Robledo, C.; Mnnist, T.; Grantz, K.L. Air pollution exposure and preeclampsia among US women with and without asthma. Environ. Res. 2016, 148, 248-255. [CrossRef]

7. Mendola, P.; Wallace, M.; Hwang, B.S.; Liu, D.; Robledo, C.; Männistö, T.; Sundaram, R.; Sherman, S.; Ying, Q.; Grantz, K.L. Preterm birth and air pollution: Critical windows of exposure for women with asthma. J. Allergy Clin. Immunol. 2016, 138, 432-440. [CrossRef]

8. Seeni, I.; Ha, S.; Nobles, C.; Liu, D.; Sherman, S.; Mendola, P. Air pollution exposure during pregnancy: Maternal asthma and neonatal respiratory outcomes. Ann. Epidemiol. 2018, 28, 612-618. [CrossRef]

9. Zhu, Y.; Zhang, C.; Liu, D.; Ha, S.; Kim, S.S.; Pollack, A.; Mendola, P. Ambient Air Pollution and Risk of Gestational Hypertension. Am. J. Epidemiol. 2017, 186, 334-343. [CrossRef]

10. Klepac, P.; Locatelli, I.; Korosec, S.; Kunzli, N.; Kukec, A. Ambient air pollution and pregnancy outcomes: A comprehensive review and identification of environmental public health challenges. Environ. Res. 2018, 167, 144-159. [CrossRef]

11. Mendola, P.; Ha, S.; Pollack, A.Z.; Zhu, Y.; Seeni, I.; Kim, S.S.; Sherman, S.; Liu, D. Chronic and Acute Ozone Exposure in the Week Prior to Delivery Is Associated with the Risk of Stillbirth. Int. J. Environ. Res. Public Health 2017, 14, 731. [CrossRef] [PubMed]

12. Hu, H.; Ha, S.; Roth, J.; Kearney, G.; Talbott, E.O.; Xu, X. Ambient Air Pollution and Hypertensive Disorders of Pregnancy: A Systematic Review and Meta-analysis. Atmos. Environ. (1994) 2014, 97, 336-345. [CrossRef] [PubMed]

13. Pedersen, M.; Stayner, L.; Slama, R.; Sørensen, M.; Figueras, F.; Nieuwenhuijsen, M.J.; Raaschou-Nielsen, O.; Dadvand, P. Ambient air pollution and pregnancy-induced hypertensive disorders: A systematic review and meta-analysis. Hypertension 2014, 64, 494-500. [CrossRef] [PubMed]

14. Ha, S.; Sundaram, R.; Buck Louis, G.M.; Nobles, C.; Seeni, I.; Sherman, S.; Mendola, P. Ambient air pollution and the risk of pregnancy loss: A prospective cohort study. Fertil. Steril. 2018, 109, 148-153. [CrossRef]

15. Yuan, L.; Zhang, Y.; Gao, Y.; Tian, Y. Maternal fine particulate matter (PM2.5) exposure and adverse birth outcomes: An updated systematic review based on cohort studies. Environ. Sci. Pollut. Res. Int. 2019, 26, 13963-13983. [CrossRef] 
16. Dewulf, B.; Neutens, T.; Lefebvre, W.; Seynaeve, G.; Vanpoucke, C.; Beckx, C.; Van de Weghe, N. Dynamic assessment of exposure to air pollution using mobile phone data. Int. J. Health Geogr. 2016, 15, 14. [CrossRef]

17. Klepeis, N.E.; Nelson, W.C.; Ott, W.R.; Robinson, J.P.; Tsang, A.M.; Switzer, P.; Behar, J.V.; Hern, S.C.; Engelmann, W.H. The National Human Activity Pattern Survey (NHAPS): A resource for assessing exposure to environmental pollutants. J. Expo. Anal. Environ. Epidemiol. 2001, 11, 231-252. [CrossRef] [PubMed]

18. Bulletin, A.P. Asthma in Pregnancy. Clinical management guidelines for obstetrician-gynecologists. Obstet. Gynecol. 2008, 111, 457-464.

19. RTI. Miniaturized, Wearable Device Gathers Real-Time Data to Support Environmental and Occupational Health Studies. 2020. Available online: https://www.rti.org/impact/micropem-sensor-measuring-exposureair-pollution (accessed on 23 April 2020).

20. Williams, R.; Kaufman, A.; Hanley, T.; Rice, J.; Garvey, S. Evaluation of Field-Deployed Low Cost PM Sensors; U.S. Environmental Protection Agency: Washington, DC, USA, 2014.

21. Barzyk, T.; Huang, H.; Williams, R.; Kaufman, A.; Essoka, J. Advice and Frequently Asked Questions (FAQs) for Citizen-Science Environmental Health Assessments. Int. J. Environ. Res. Public Health 2018, 15, 960. [CrossRef]

22. Wheeler, A.J.; Xu, X.; Kulka, R.; You, H.; Wallace, L.; Mallach, G.; Ryswyk, K.V.; MacNeill, M.; Kearney, J.; Rasmussen, P.E.; et al. Windsor, Ontario exposure assessment study: Design and methods validation of personal, indoor, and outdoor air pollution monitoring. J. Air Waste Manag. Assoc. 2011, 61, 142-156. [CrossRef]

23. Cho, S.; Chartier, R.; Mortimer, K.; Dherani, M.; Tafatatha, T. A Personal Particulate Matter Exposure Monitor to Support Household Air Pollution Exposure and Health Studies. In Proceedings of the IEEE Global Humanitarian Technology Conference (GHTC), Seattle, WA, USA, 13-16 October 2016.

24. EPA. NAAQS Table. 2016. Available online: https://www.epa.gov/criteria-air-pollutants/naaqs-table (accessed on 6 December 2019).

25. Simons, E.; Dell, S.D.; Moineddin, R.; To, T. Neighborhood Material Deprivation Is Associated with Childhood Asthma Development: Analysis of Prospective Administrative Data. Can. Respir. J. 2019, 2019, 6808206. [CrossRef] [PubMed]

26. Arnetz, B.B.; Arnetz, J.; Harkema, J.R.; Morishita, M.; Slonager, K.; Sudan, S.; Jamil, H. Neighborhood air pollution and household environmental health as it relates to respiratory health and healthcare utilization among elderly persons with asthma. J. Asthma 2019, 57,1-12. [CrossRef]

27. O'Lenick, C.R.; Winquist, A.; Mulholland, J.A.; Friberg, M.D.; Chang, H.H.; Kramer, M.R.; Darrow, L.A.; Sarnat, S.E. Assessment of neighbourhood-level socioeconomic status as a modifier of air pollution-asthma associations among children in Atlanta. J. Epidemiol. Community Health 2017, 71, 129-136. [CrossRef]

28. Wilhelm, M.; Qian, L.; Ritz, B. Outdoor air pollution, family and neighborhood environment, and asthma in LA FANS children. Health Place 2009, 15, 25-36. [CrossRef] [PubMed]

29. Mohajerani, A.; Bakaric, J.; Jeffrey-Bailey, T. The urban heat island effect, its causes, and mitigation, with reference to the thermal properties of asphalt concrete. J. Environ. Manag. 2017, 197, 522-538. [CrossRef] [PubMed]

30. Heaviside, C.; Macintyre, H.; Vardoulakis, S. The Urban Heat Island: Implications for Health in a Changing Environment. Curr. Environ. Health Rep. 2017, 4, 296-305. [CrossRef] [PubMed]

31. Murphy, V.E.; Jensen, M.E.; Gibson, P.G. Asthma during Pregnancy: Exacerbations, Management, and Health Outcomes for Mother and Infant. Semin. Respir. Crit. Care Med. 2017, 38, 160-173.

32. Bozkurt, Z.; Dogan, G.; Arslanbas, D.; Pekey, B.; Pekey, H.; Dumanoğlu, Y.; Bayram, A.; Tuncel, G. Determination of the personal, indoor and outdoor exposure levels of inorganic gaseous pollutants in different microenvironments in an industrial city. Environ. Monit. Assess. 2015, 187, 590. [CrossRef]

33. Weisel, C.P.; Zhang, J.; Turpin, B.J.; Morandi, M.T.; Colome, S.; Stock, T.H.; Spektor, D.M.; Korn, L.; Winer, A.M.; Kwon, J.; et al. Relationships of Indoor, Outdoor, and Personal Air (RIOPA). Part I. Collection methods and descriptive analyses. Res. Rep. Health Eff. Inst. 2005, 130 Pt 1, 1-107.

34. Gold, D.R.; Allen, G.; Damokosh, A.; Serrano, P.; Hayes, C.; Castiilejos, M. Comparison of Outdoor and Classroom Ozone Exposures for School Children in Mexico City. J. Air Waste Manag. Assoc. 1996, 46, 335-342. 
35. Ouidir, M.; Giorgis-Allemand, L.; Lyon-Caen, S.; Morelli, X.; Cracowski, C.; Pontet, S.; Pin, I.; Lepeule, J.; Siroux, V.; Slama, R. Estimation of exposure to atmospheric pollutants during pregnancy integrating space-time activity and indoor air levels: Does it make a difference? Environ. Int. 2015, 84, 161-173. [CrossRef] [PubMed]

36. Han, B.; Hu, L.W.; Bai, Z. Human Exposure Assessment for Air Pollution. Adv. Exp. Med. Biol. 2017, 1017, 27-57. [PubMed]

37. Lawless, P.; Thornburg, J.; Rodes, C.; Williams, R. Personal exposure monitoring wearing protocol compliance: An initial assessment of quantitative measurement. J. Expo. Sci. Environ. Epidemiol. 2012, 22, 274-280. [CrossRef] [PubMed]

38. Nethery, E.; Brauer, M.; Janssen, P. Time-activity patterns of pregnant women and changes during the course of pregnancy. J. Expo. Sci. Environ. Epidemiol. 2009, 19, 317-324. [CrossRef]

39. Zielonka, T.; Zycinska, K. Are physicians specialists on the impact of air pollution on health? Eur. Respir. J. 2016, 48, PA4288.

40. Mirabelli, M.C.; Damon, S.A.; Beavers, S.F.; Sircar, K.D. Patient-Provider Discussions About Strategies to Limit Air Pollution Exposures. Am. J. Prev. Med. 2018, 55, e49-e52. [CrossRef]

41. Powell, P.; Brunekreef, B.; Grigg, J. How do you explain the risk of air pollution to your patients? Breathe (Sheff) 2016, 12, 201-203. [CrossRef]

42. Ramirez, A.S.; Ramondt, S.; Van Bogart, K.; Perez-Zuniga, R. Public Awareness of Air Pollution and Health Threats: Challenges and Opportunities for Communication Strategies to Improve Environmental Health Literacy. J. Health Commun. 2019, 24, 75-83. [CrossRef]

43. Lovinsky-Desir, S.; Lawrence, J.; Jung, K.H.; Rundle, A.G.; Hoepner, L.A.; Yan, B.; Perera, F.; Perzanowski, M.S.; Miller, R.L.; Chillrud, S.N. Assessment of exposure to air pollution in children: Determining whether wearing a personal monitor affects physical activity. Environ. Res. 2018, 166, 340-343. [CrossRef]

(C) 2020 by the authors. Licensee MDPI, Basel, Switzerland. This article is an open access article distributed under the terms and conditions of the Creative Commons Attribution (CC BY) license (http://creativecommons.org/licenses/by/4.0/). 